\title{
A clinical and electron microscopic study of a calcifying epithelial odontogenic tumour
}

\author{
A. R. MAINWARING, ALI AHMED, J. M. HOPKINSON, AND P. ANDERSON \\ From the Departments of Pathology and Otolaryngology, University of Manchester, and Department of \\ Pathology, Bradford Royal Infirmary
}

SYNOPSIS The clinical features and the histological and ultrastructural appearances of a case of calcifying epithelial odontogenic tumour are described. The ultrastructural features of the cells of this tumour are comparable to cells of the stratum intermedium of the enamel organ. It is suggested that the amyloid-staining material found in this tumour is a cellular secretion rather than a cytoplasmic degeneration.

The calcifying epithelial odontogenic tumour was first described as a distinct entity by Pindborg (1958) and is believed to originate in the reduced enamel organ or the oral epithelium. Evans (1966) has suggested that the tumour is a variant of ameloblastoma, but, as Abrams and Howell (1967) have pointed out the calcifying epithelial odontogenic tumour differs from it by having no preameloblasts or stellate reticulum.

Vap, Dahlin, and Turlington (1970) in the most recent review of the literature have indicated a total number of 43 reported cases which included their report on eight cases from the files of the Mayo Clinic.

The calcifying epithelial odontogenic tumour is a tumour with no sex predilection and is usually found in the molar or premolar regions. It has been found in the mandible twice as often as in the maxilla. The tumour has also been observed in an extraosseous situation on three occasions (Pindborg, 1966; Abrams and Howell, 1967; Patterson, Martin, DeJean, and Burzynski, 1969). It is a slow growing, locally invasive tumour and there has been no report of distant metastases. The tumours of intra-osseous origin have frequently been associated with an unerupted tooth, though Vap et al (1970) indicated that this had been established in only 18 of the $\mathbf{4 0}$ cases of intra-osseous tumour.

\section{Case Report}

The material used in this study was obtained in September 1969 from a male patient who was first seen in 1953 at the age of 45 years in Bradford Royal Infirmary. His complaint at that time was of a Received for publication 14 July 1970. lump in the region of the left maxillary tuberosity (Fig. 1) which had been present for 18 months and had greatly increased in size during the previous six $\vec{y}$

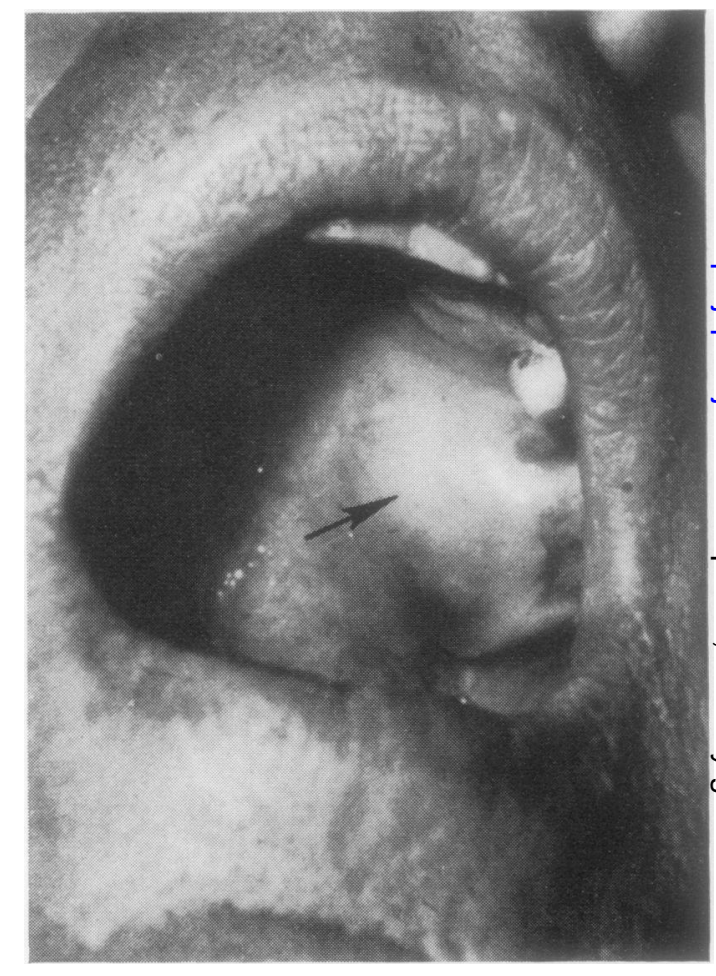

Fig. 1. Swelling (arrowed) in region of left maxillary tuberosity. 


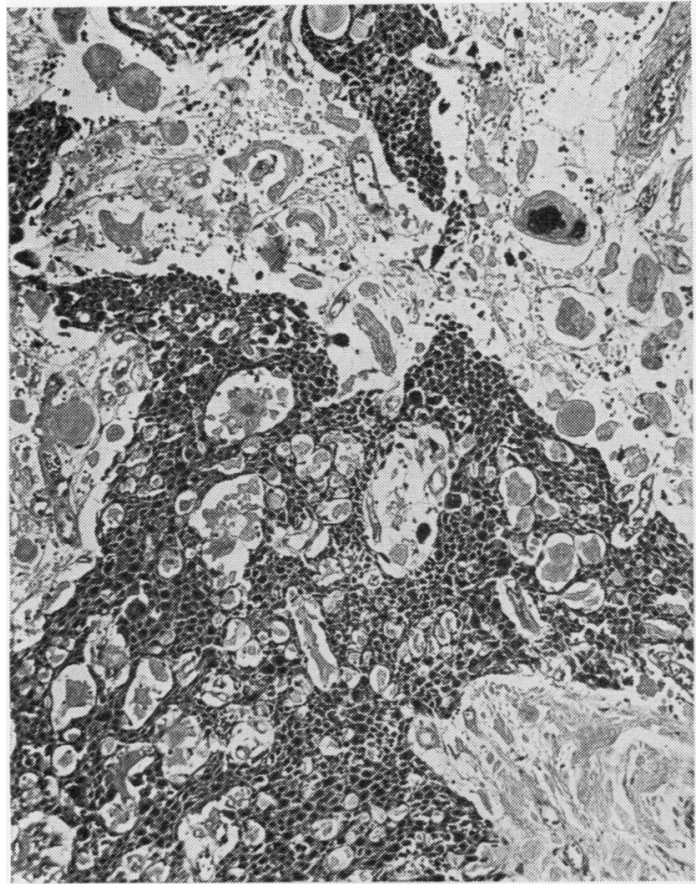

Fig. 2.

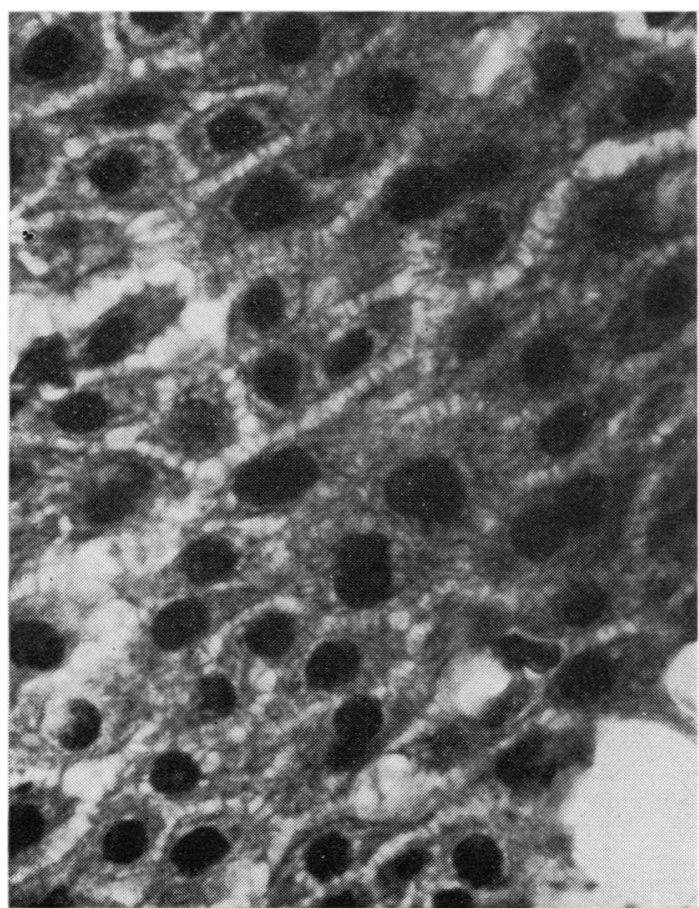

Fig. 3.

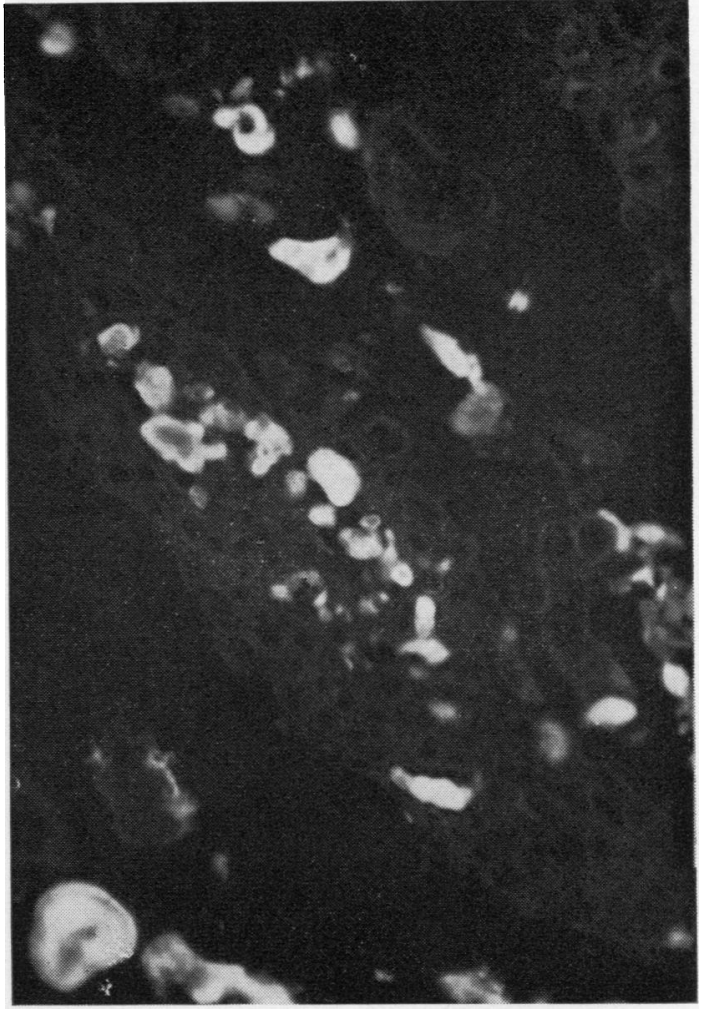

Fig. 4.

Fig. 2. Mass of tumour cells with intracytoplasmic and stromal homogeneous substance. One focus of calcification in the stromal homogeneous substance in top right hand corner (haematoxylin and eosin $\times 80$ ).

Fig. 3. Tumour cells with intercellular bridges (haematoxylin and eosin $\times 800$ ).

Fig. 4. Fluorescence of homogeneous substance under ultraviolet light (thioflavine $T \times 300$ ). 
months. A radiograph taken in 1953 was reported as follows: 'appearances are consistent with neoplastic change in the left upper jaw in the region of the sixth or seventh molars. There is a large unerupted tooth in relation to this mass. The floor of the left antrum appears to be intact.' The lump was excised and a resulting defect in the hard palate was closed by a dental plate. No definitive diagnosis was made at that time when it was considered to be a form of adamantinoma or a salivary carcinoma. The sections

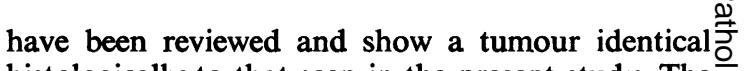
histologically to that seen in the present study. The patient received postoperative radiotherapy and was followed up at regular intervals. There was nosign of recurrence of the tumour until July 1969, 등 when the patient complained of ill-fitting dentures $\overline{\bar{s}}$ and examination showed a painless, non-ulcerated $\overrightarrow{\widetilde{\alpha}}$

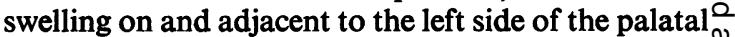
defect. This was subsequently excised and sent for ${ }^{\mathrm{C}}$ histological examination.

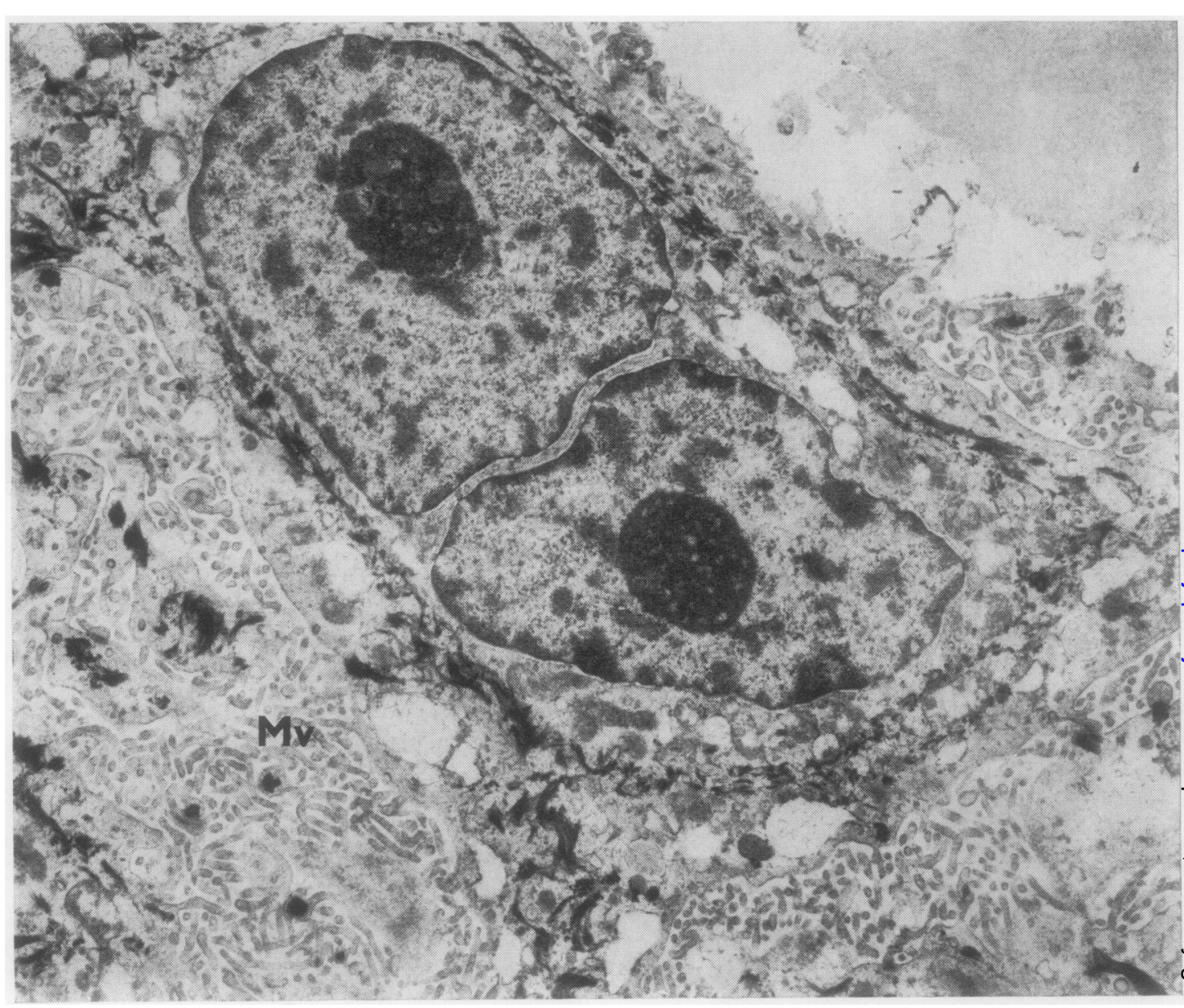

Fig. 5. Binucleate tumour cell with prominent nucleoli. Numerous microvilli $(\mathrm{Mv}) \times 20,000$. 


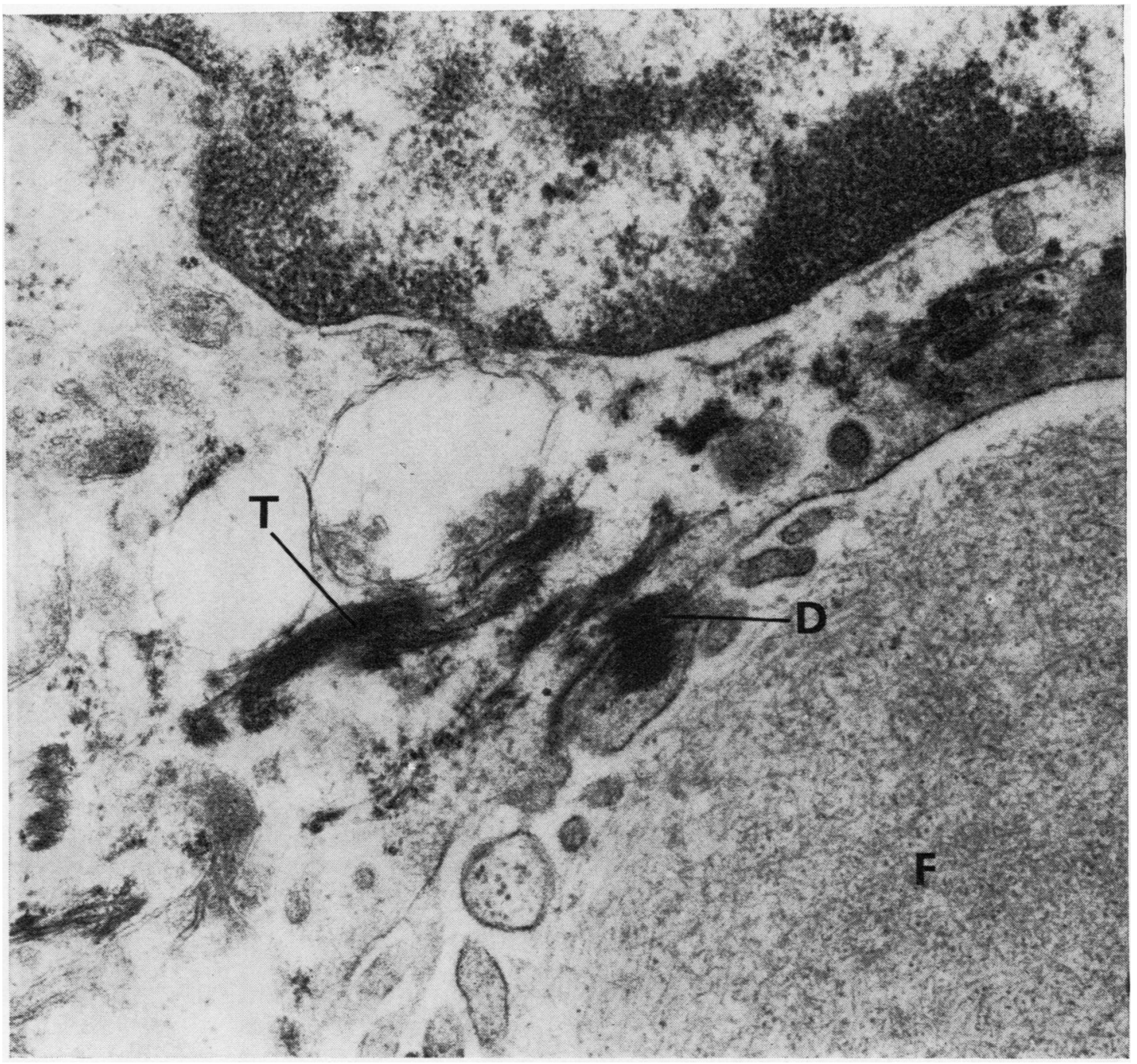

Fig. 6. Part of a tumour cell to show tonofilaments (T) and desmosome (D). Stromal microfibrils (F) in lower right hand corner $\times 60,000$. 


\section{Material and Methods}

The specimen consisted of a firm, light brown nodule, $2 \mathrm{~cm}$ diameter, covered on one aspect by intact mucosa. It was received in formaldehyde. Small blocks of tissue from the margin of the nodule were postfixed in osmium tetroxide in phosphate buffer and embedded in araldite. Ultra-thin sections were stained with aqueous uranyl acetate and Reynold's lead citrate and examined under the electron microscope. Other blocks were embedded in paraffin and sections stained with haematoxylin and eosin, van Gieson, PAS technique, Gordon and $\stackrel{0}{\vec{*}}$

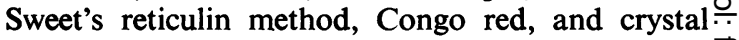
violet for routine microscopy. Unstained sections $\overrightarrow{\vec{F}}$ and those stained with thioflavine $\mathrm{T}$ were examined under ultraviolet light. Congo-red stained sections were also examined under polarized light.

\section{Results}

\section{LIGHT MICROSCOPY}

The tumour was composed of closely packed polyhedral cells and abundant stroma composed mainly

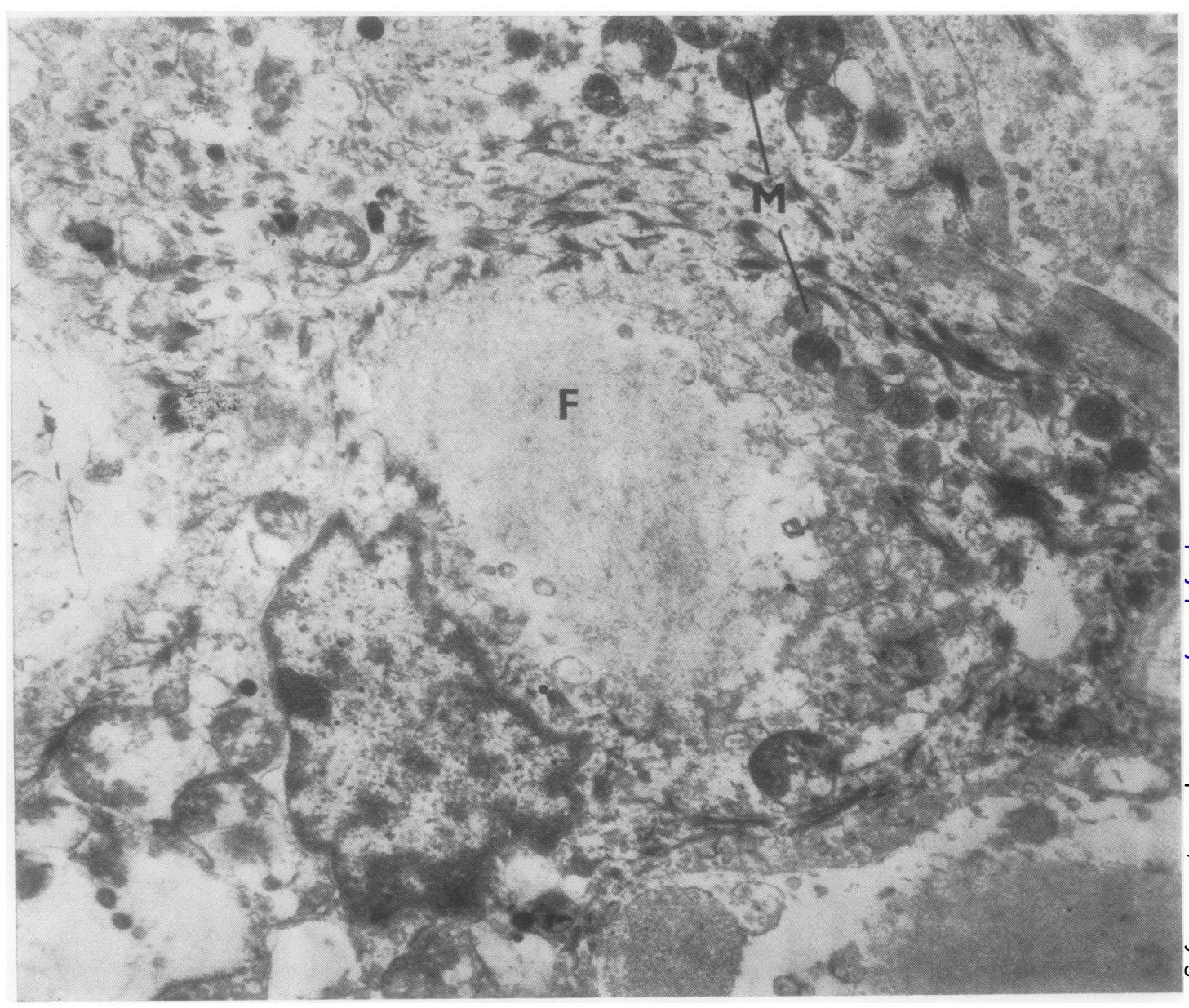

N 
of a homogeneous substance as shown in Figure 2. In the cellular areas there was some degree of nuclear pleomorphism but no mitotic activity. Intercellular bridges were seen in some areas (Fig. 3). Many of the cells were enlarged and their nuclei displaced and distorted by intracytoplasmic homogeneous substance. At the borders of the cell masses and also lying free in the stroma were several multinucleate cells. Calcification of the stromal homogeneous substance was present (Fig. 2) but by no means a prominent feature. The homogeneous substance reacted metachromatically with crystal violet; it showed variable affinity for Congo red and a dichroic effect when examined under polarized light; in unstained sections and those stained with thioflavine $T$ it gave a greenish fluorescence with ultraviolet light (Fig. 4). Reticulin preparations showed a coarse reticulin network enveloping the homogeneous substance. Only scanty amounts of mature collagen were seen.

\section{ELECTRON MICROSCOPY}

The tumour cells were large and polyhedral. The plasma membranes showed many folds and micro-

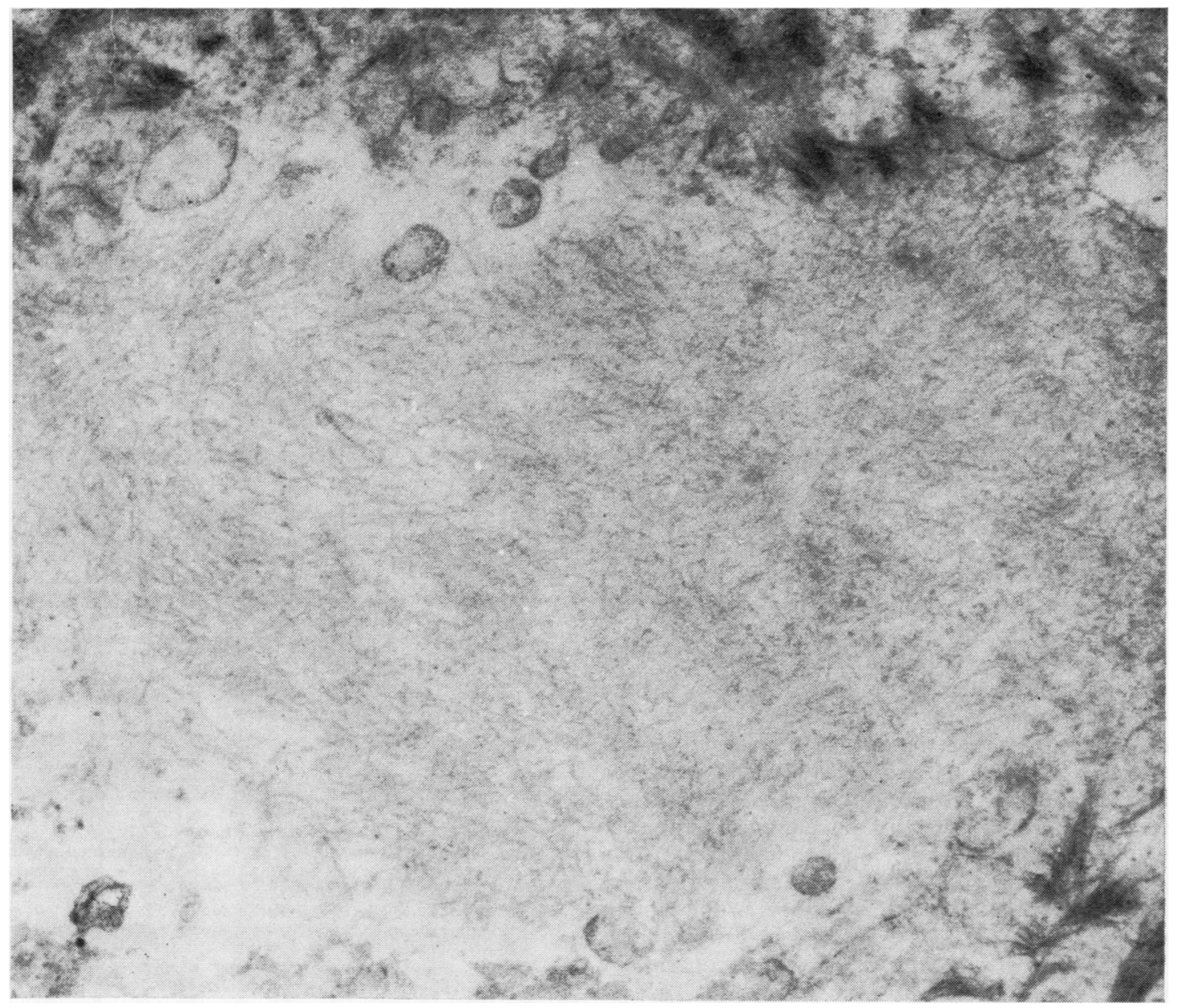

Fig. 8. Higher magnification of intracytoplasmic fibrillary material $\times 60,000$. 
villous projections were particularly prominent (Fig. 5). The cell nuclei were round or ovoid and some contained prominent nucleoli. Occasional cells were binucleate (Fig. 5). The cytoplasm was abundant and contained numerous mitochondria some of which were large and empty (Fig. 7) and many tonofilaments which were arranged at irregular angles and seen to terminate at the intercellular bridges where cell attachment was provided by desmosomes (Fig. 6). Rough endoplasmic reticulum and occasional Golgi bodies were seen. The intracytoplasmic homogeneous substance was composed of microfibrils approximately $50 \AA$ in diameter with no obvious periodicity. These were situated in the region of the Golgi apparatus and surrounded by several vesicles (Figs. 7 and 8). The extracellular homogeneous substance was composed mainly of microfibrils of similar size and nature as the cytoplasmic microfibrils though some large collagen fibres were also seen.

\section{Discussion}

The tumour presented in this report fulfils the criteria of calcifying epithelial odontogenic tumour both in its morphological features and clinical behaviour. The ultrastructural features of the tumour cells were recently described by Anderson, Kim, and Minkowitz (1969) and we can confirm their findings. The cells show features commonly seen in epidermal cells in keeping with origin from the enamel organ or oral epithelium. In a study of the histogenesis of this tumour Gon (1965) stated that the cell type was like that of the stratum spinosum of the epidermis and comparable to the cells of the stratum intermedium of the enamel organ. The ultrastructural features of the cells in this tumour certainly compare well with the cells of the stratum intermedium (Pannesse, 1960). Vickers, Dahlin, and Gorlin (1965) and Ranløv and Pindborg (1966) demonstrated amyloid-staining material within the tumour cells and in the surrounding stroma and regarded it as a degeneration of the cytoplasm of the cells with subsequent affinity for mineral salts. However, the question whether the amyloid-staining material might represent an attempt at matrix formation destined for calcification was raised by Gon (1965). In this example of the tumour the demonstration of intracytoplasmic microfibrils in the region of the Golgi apparatus surrounded by vesicles possibly of a secretory nature gives support to Gon's hypothesis.

The exact nature of the intracytoplasmic and stromal microfibrils of approximately $50 \AA$ is debatable. Anderson et al (1969) argued that they might represent basal lamina. Despite giving the histo- $\overrightarrow{\vec{D}}$ chemical reactions of amyloid, the fibre size is smallerthan that of classical amyloid (Sorenson and Shima-듬 mura, 1964). Vassar and Culling (1961), referring to the amyloid-like material found in medullary $\Phi$ carcinoma of the thyroid, postulated that its staining properties resulted from the presence of high levels of acid mucopolysaccharide in the neoplastic secre- $\vec{\circ}$ tions of thyroglobulin glycoprotein. Various glyco- $\overrightarrow{\mid}$ protein complexes, which have been demonstrated ${ }_{\Omega}^{\omega}$ in the enamel organ (Reith and Butcher, 1967), will흥 accept the classical amyloid stains. We therefore suggest that the cell products of the tumour we describe may represent a comparable glycoprotein? and thus account for its staining properties.

We wish to thank Mr K. Harrison for his permission to publish the clinical details of this case and $\mathrm{Mr} z$ G. C. W. Humberstone and Miss J. McConville for technical assistance.

\section{References}

Abrams, A. M., and Howell, F. V. (1967). Calcifying epithelial odontogenic tumors; report of four cases. J. Amer. dent. Ass., 74, 1231-1240.

Anderson, H. C., Kim, B., and Minkowitz, S. (1969). Calcifying epithelial odontogenic tumor of Pindborg. An electron microscopic study. Cancer (Philad.), 24, 585-596.

Evans, R. W. (1966). Histological Appearances of Tumours, 2nd ed., $\overrightarrow{\vec{D}}$ p. 972. Livingstone, Edinburgh.

Gon, F. (1965). The calcifying epithelial odontogenic tumour. Report 3 of a case and a study of its histogenesis. Brit. J. Cancer, 19, 39-50.

Pannesse, E. (1960). Observations on the ultrastructure of the enamel organ. Part 1. Stellate reticulum and stratum intermedium. $\frac{\bar{O}}{0}$

Patterson, J. T., Martin, T. H., DeJean, E. K., and Burzynski, N. J. ס (1969). Extraosseous calcifying epithelial odontogenic tumor. 3 Oral Surg., 27, 363-367.

Pindborg, J. J. (1958). A calcifying epithelial odontogenic tumor. O Cancer (Philad.), 11, 838-843.

Pindborg, J. J. (1966). The calcifying epithelial odontogenic tumour. Review of the literature and report of an extraosseous case. 음 Acta odont. scand., 24, 419-430.

Ranløv, P., and Pindborg, J. J. (1966). The amyloid nature of the $\frac{D}{O}$ homogeneous substance in the calcifying epithelial odontogenic tumour. Acta path. microbiol. scand., 68, 169-174.

Reith, E. J., and Butcher, E. O. (1967). In Structural and Chemical o Organization of Teeth, edited by A. E. W. Miles, p. 385 . Academic Press, New York and London.

Sorenson, G. D., and Shimamura, T. (1964). Experimental amyloidosis. III. Light and electron microscopic observations of $\omega$ renal glomeruli. Lab. Invest., 13, 1409-1417.

Vap, D. R., Dahlin, D. C., and Turlington, E. G. (1970). Pindborg tumour; the so-called calcifying epithelial odontogenic tumour. Cancer (Philad.), 25, 629-636.

Vassar, P. S., and Culling, C. F. (1961). The significance of amyloid $\mathscr{D}$ in carcinoma of the thyroid gland. Amer. J. clin. Path., 36, 244-247.

Vickers, R. A., Dahlin, D. C., and Gorlin, R. J. (1965). Amyloidcontaining odontogenic tumours. Oral Surg., 20, 476-480. 